\title{
Editorial
}

Oncology

\section{Beyond the Basics: The Differential Effects of Demographics and Hepatitis Status on Treatment Outcome in Hepatocellular Carcinoma}

\author{
Ahmed O. Kaseb \\ Department of Gastrointestinal Medical Oncology, The University of Texas MD Anderson Cancer Center, \\ Houston, Tex., USA
}

Hepatocellular carcinoma (HCC) is the most common primary neoplasm of the liver, accounting for about 700,000 newly diagnosed patients per year, and more than two thirds of new HCC cases are from the Asia-Pacific (AP) region [1], mainly linked to chronic hepatitis $\mathrm{B}$ infection. Although the incidence of HCC is particularly high in parts of Asia and sub-Saharan Africa, recent studies have reported a clear rise in the number of cases in Western Europe and the United States, largely because of the increase in hepatitis-C-related liver disease [2]. Unfortunately, most patients with HCC are not candidates for any curative treatments because of advanced disease at presentation and/or a background of advanced chronic liver disease. Furthermore, the growth of HCC depends on stimulatory effects of various growth factors, which bind to tyrosine kinase receptors and hence activate various intracellular signaling pathways which subsequently lead to tumor cell proliferation, survival, migration and metastasis. However, HCC is an angiogenesis-driven tumor, characterized by proangiogenic cytokine upregulation, hypervascularity and early vascular invasion.

The current study [3] accompanying this editorial reports on an Asian cohort of 51 patients with advanced HCC who received the intravenous monoclonal antibody, bevacizumab, that binds to circulating vascular en- dothelial growth factor (VEGF)-A ligand and erlotinib, an oral tyrosine kinase inhibitor of the epithelial growth factor receptor. Of note, VEGF and epidermal growth factor pathways share a common downstream cascade of events and eventually stimulate angiogenesis signaling [4-6]. Additionally, upregulation of VEGF has been implicated in resistance to epithelial growth factor receptor inhibition [7]. Recent studies, including ours, indicated a signal of activity of both agents in HCC [8-12]. The authors report a progression-free survival at 16 weeks (PFS16 ) of $35.3 \%$ [95\% confidence interval (CI) 22.4-49.9], a median PFS of 2.9 months (95\% CI 1.3-4.42) and a median overall survival (OS) of 10.7 months (95\% CI 6.215.2 ) while grade $3 / 4$ adverse events were not common. Results from our recent study [8] of the same combination in patients with advanced HCC in the US showed an overall higher magnitude of benefit with PFS-16 of $64 \%$ (95\% CI 51-76), a median PFS of 7.2 months (95\% CI 5.6-8.3) and a median OS of 13.7 months (95\% CI 9.619.7). Notably, there was a significant difference between the incidence of hepatitis B virus (HBV) and hepatitis C virus (HCV) infections in both cohorts: 17 and 29\% in our US cohort as compared to 82 and $8 \%$ in the Asian study, respectively [3].

\section{KARGER}

E-Mail karger@karger.com www.karger.com/ocl
(C) 2013 S. Karger AG, Basel

0030-2414/13/0851-0041\$38.00/0
Ahmed O. Kaseb, MD

Department of Gastrointestinal Medical Oncology, Unit 426

The University of Texas MD Anderson Cancer Center

1515 Holcombe Blvd, Houston, TX 77030 (USA)

E-Mail akaseb@mdanderson.org 
These observations of the differential outcome based on demographics, geographical distribution and hepatitis status in patients with HCC receiving systemic therapy are consistent with similar observations in patients with HCC treated with transarterial chemoembolization (TACE) from the two randomized TACE studies, conducted in Europe [13] and Asia [14], which established TACE as a standard therapeutic modality to treat unresectable HCC. Llovet et al. [13] reported a European population with an $87 \% \mathrm{HCV}$ infection rate, while Lo et al. [14] reported an Asian population with an $80 \% \mathrm{HBV}$ infection rate. The European study reported a 1- and 2-year survival incidence of 82 and 63 versus 63 and 27\%, as compared with those who received placebo, whereas the Asian study [14] reported a 1- and 2-year survival incidence of 57 and 31 versus 32 and $11 \%$, as compared with those who received placebo. Furthermore, similar observations were recently reported in patients treated with sorafenib, the current standard of care systemic therapy for patients with advanced HCC. The phase III study in 602 patients with HCC (SHARP, Sorafenib HCC Assessment Randomized Protocol), was the first international, randomized, double-blind, placebo-controlled systemic therapy study to demonstrate statistically significant improvement in OS in nonsurgical patients with HCC [15]. Median OS was 10.7 months in the sorafenib group and 7.9 months in the placebo group; the hazard ratio (HR) in the sorafenib group was 0.69 (95\% CI 0.55-0.87; p < 0.001 ). The median time to progression (TTP) was 5.5 months in the sorafenib group and 2.8 months in the placebo group $(\mathrm{p}<0.001)$. Another phase III study of sorafenib in the AP region showed improved median OS ( 6.5 vs. 4.2 months; $\mathrm{p}=0.014)$ and TTP ( 2.8 vs. 1.4 months; $\mathrm{p}=0.0005)$, compared with placebo. Most recently, two studies of exploratory subgroup analyses of data from both phase III studies of sorafenib (SHARP and AP trials) were reported and showed differential outcome in patients based on demographics and hepatitis status. The subset analysis of the SHARP study showed that sorafenib consistently improved median OS compared with placebo, as reflected by HRs of $0.50-0.85$, and consistently improved the median TTP (HR 0.40-0.64), except in HBVpositive patients (HR 1.03) [16]. Furthermore, HCV-infected patients had a longer median OS (14.0 vs. 7.4 months) and TTP (7.6 vs. 2.8 months) as compared with those who received placebo, while HBV-infected patients had a longer median OS (9.7 vs. 6.1 months) but a shorter median TTP (2.7 vs. 4.2 months) as compared with those who received placebo. However, the HBV subset in the SHARP study was much smaller than in the other sub- sets and had a poorer performance status. Interestingly, subset analyses from the AP trial showed that HBV-infected patients had a longer median OS (5.9 vs. 4.1 months) and TTP (2.7 vs. 1.4 months) compared with placebo, while these outcome measures were notably shorter than in those patients with HBV from the SHARP study. While direct comparison is not possible, this notable difference in outcome may reflect the difference in demographics and geographical distribution between patient populations of the SHARP and AP sorafenib trials even in patients with the same risk factor (HBV infection).

In summary, observations from the study of Hsu et al. [3] include an overall lower magnitude of outcome measures as compared to the outcome reported by our recent study [8] using the same systemic therapy combination. Those observations are consistent with other pivotal studies of systemic and local therapies for patients with unresectable HCC and advocate for the need to assess results from HCC clinical trials in this context with respect to specific demographics and HCC risk factors. Thus, this differential outcome using the same therapy modality and agents could be explained based on the markedly heterogeneous patient population with regard to demographics, geographical distribution and risk factors including hepatitis status. Therefore, future HCC clinical trials should be designed and powered to address the need for major prognostic stratification refinement based on demographics, geographical distribution of patients and hepatitis status. This approach should become the standard stratification criterion which may help in reducing the noted heterogeneity in populations randomized into HCC clinical trials and may decrease futility rates among investigational drug trials.

\section{Disclosure Statement}

I have no commercial associations (e.g., consultancies, stock ownership, equity interests, or patent-licensing arrangements) that might pose a conflict of interest in connection with the submitted article.
Kaseb 


\section{References}

$>1$ Srivatanakul P, Sriplung H, Deerasamee S: Epidemiology of liver cancer: an overview. Asian Pac J Cancer Prev 2004;5:118-125.

$>2$ El-Serag HB, Mason AC: Rising incidence of hepatocellular carcinoma in the United States. N Engl J Med 1999;340:745-750.

$>3$ Hsu CH, Kang YK, Yang TS, Shun CT, Shao YY, Su WC, Sandoval-Tan J, Chiou TJ, Jin K, Hsu C, Cheng AL: Bevacizumab with erlotinib as first-line therapy in Asian patients with advanced hepatocellular carcinoma: a multicenter phase II study. Oncology 2013; 85:44-52.

4 Hirata A, Ogawa S, Kometani T, et al: ZD1839 (Iressa) induces antiangiogenic effects through inhibition of epidermal growth factor receptor tyrosine kinase. Cancer Res 2002; 62:2554-2560.

$>5$ Tamesa T, Iizuka N, Mori N, et al: High serum levels of vascular endothelial growth factor after hepatectomy are associated with poor prognosis in hepatocellular carcinoma. Hepatogastroenterology 2009;56:1122-1126.
6 Giannelli G, Sgarra C, Porcelli L, Azzariti A, Antonaci S, Paradiso A: EGFR and VEGFR as potential target for biological therapies in HCC cells. Cancer Lett 2008;262:257-264.

7 Moller S, Becker U: Insulin-like growth factor 1 and growth hormone in chronic liver dis ease. Dig Dis 1992;10:239-248.

8 Kaseb AO, Garrett-Mayer E, Morris JS, et al: Efficacy of bevacizumab plus erlotinib for advanced hepatocellular carcinoma and predictors of outcome: final results of a phase II trial. Oncology 2012;82:67-74.

-9 Thomas MB, Morris JS, Chadha R, et al: Phase II trial of the combination of bevacizumab and erlotinib in patients who have advanced hepatocellular carcinoma. J Clin Oncol 2009; 27:843-850.

10 Siegel AB, Cohen EI, Ocean A, et al: Phase II trial evaluating the clinical and biologic effects of bevacizumab in unresectable hepatocellular carcinoma. J Clin Oncol 2008;26: 2992-2998.

11 Thomas MB, Chadha R, Glover K, et al: Phase 2 study of erlotinib in patients with unresectable hepatocellular carcinoma. Cancer 2007; 110:1059-1067.
12 Philip PA, Mahoney MR, Allmer C, et al: Phase II study of erlotinib (OSI-774) in patients with advanced hepatocellular cancer. J Clin Oncol 2005;23:6657-6663.

13 Llovet JM, Real MI, Montana X, et al: Arterial embolisation or chemoembolisation versus symptomatic treatment in patients with unresectable hepatocellular carcinoma: a randomised controlled trial. Lancet 2002;359: 1734-1739.

14 Lo CM, Ngan H, Tso WK, et al: Randomized controlled trial of transarterial lipiodol chemoembolization for unresectable hepatocellular carcinoma. Hepatology 2002;35:11641171.

15 Llovet JM, Ricci S, Mazzaferro V, et al: Sorafenib in advanced hepatocellular carcinoma. N Engl J Med 2008;359:378-390.

16 Bruix J, Raoul JL, Sherman M, et al: Efficacy and safety of sorafenib in patients with advanced hepatocellular carcinoma: subanalyses of a phase III trial. J Hepatol 2012;57:821829. 